\title{
A VISÃO DO PROFISSIONAL ENFERMEIRO SOBRE O TRATAMENTO DA HANSENÍASE NA ATENÇÃO BÁSICA
}

\author{
The nurse's view on Leprosy treatment in primary health care
}

\section{La visión del profesional enfermero sobre el tratamiento de la lepra en la atención básica}

\author{
Mara Dayanne Alves Ribeiro \\ Universidade Federal do Ceará - UFC - Sobral (CE) - Brasil \\ Iraneide da Silva Castillo \\ Empresa Maranhense de Serviços Hospitalares - EMSERH - São Luis - (MA) - Brasil
}

Jefferson Carlos Araújo Silva

Hospital Universitário Presidente Dutra - São Luis - (MA) - Brasil

Sabrynna Brito Oliveira

Universidade Federal de Minas Gerais - UFMG - Belo Horizonte - Brasil

\section{RESUMO}

Objetivo: Avaliar a visão dos enfermeiros atuantes na Atenção Básica (AB) sobre o tratamento da hanseníase. Métodos: Pesquisa do tipo descritiva, com abordagem qualitativa, realizada com nove enfermeiros que trabalham no município de Cocal, Piauí, Brasil, entre os meses de janeiro a março de 2016. A coleta de dados deu-se através de questões norteadoras sobre o tratamento da hanseníase e as respostas foram transcritas e ponderadas pela análise de conteúdo. Resultados: Os enfermeiros afirmaram que a poliquimioterapia é eficaz para o tratamento da hanseníase, assim como para a redução da carga da doença e cura do paciente. A supervisão está relacionada ao tratamento correto, garantia de cura, redução de sequelas e interrupção da transmissão da doença. A falha no tratamento está associada principalmente à falta do engajamento do paciente e da família, à ausência de medicação no posto de saúde, à carência de orientação ao paciente, ao baixo nível de escolaridade e aos efeitos colaterais dos remédios. A não adesão e o abandono do tratamento foram relacionados às complicações, como o agravamento de sintomas, as incapacidades e as amputações. Conclusão: O estudo mostrou que os enfermeiros avaliados possuíam conhecimento adequado sobre aspectos do tratamento da hanseníase na $\mathrm{AB}$, evidenciando pontos que devem ser corrigidos para que se alcance excelência no combate à hanseníase, como a importância de incentivar a participação da família como coadjuvante no tratamento.

Descritores: Hanseníase; Enfermagem; Atenção Primária à Saúde; Quimioterapia Combinada.

\section{ABSTRACT}

Objective: To evaluate Primary Health Care (PHC) nurses'view on leprosy treatment. Methods: Qualitative descriptive study conducted with nine nurses working in the municipality of Cocal, Piaui, Brazil, from January to March 2016. Data were collected using guiding questions about leprosy treatment and the answers were transcribed and submitted to content analysis. Results: The nurses stated that polychemotherapy is effective in the treatment of leprosy, as well as in reducing the burden of disease and curing the patient. Supervision is related to correct treatment, guarantee of cure, reduction of sequelae and interruption of disease transmission. Treatment failure is mainly associated with patient's and family's lack of engagement, lack of medication at the health clinic, lack of patient guidance, low levels of education, and drug side effects. Noncompliance with or cessation of treatment were related to complications such as aggravation of symptoms, disabilities, and amputations. Conclusion: The study showed that the nurses evaluated have adequate knowledge about aspects of leprosy treatment in PHC, highlighting points that must be corrected in order to achieve excellence in the fight against leprosy, such as the importance of encouraging family participation as a coadjutant in the treatment.

Descriptors: Leprosy; Nursing; Primary Health Care; Drug Therapy, Combination. 


\section{RESUMEN}

Objetivo: Evaluar la visión de los enfermeros que actúan en la Atención Básica (AB) sobre el tratamiento de la lepra. Métodos: Investigación del tipo descriptiva y de abordaje cualitativo realizada con nueve enfermeros que trabajan en el municipio de Cocal, Piauí, Brasil, entre los meses de enero y marzo de 2016. La recogida de datos se dio a través de cuestiones norteadoras sobre el tratamiento de la lepra y las respuestas fueron transcritas y analizadas a través del análisis de contenido. Resultados: Los enfermeros afirmaron que la poliquimioterapia es eficaz para el tratamiento de la lepra así como para la reducción de la carga de la enfermedad y la cura del enfermo. La supervisión relacionase al tratamiento correcto, la garantía de la cura, la reducción de las secuelas y la interrupción de la trasmisión de la enfermedad. La falla del tratamiento se asocia principalmente con la falta de compromiso del paciente y de la familia, la ausencia de la medicación en el centro de salud, la carencia de orientación al paciente, el bajo nivel de escolaridad y los efectos colaterales de los remedios. La no adhesión y el abandono del tratamiento se relacionaron con las complicaciones, el agravamiento de los síntomas, las incapacidades y las amputaciones. Conclusión: El estudio mostró que los enfermeros evaluados tenían el conocimiento adecuado de los aspectos del tratamiento de la lepra en la $A B$ con evidencias de aspectos que deben ser corregidos para alcanzar la excelencia en el combate de la lepra como la importancia del incentivo a la participación de la familia como coadyuvante para el tratamiento.

Descriptores: Lepra; Enfermería; Atención Primaria de Salud; Quimioterapia Combinada.

\section{INTRODUÇÃO}

A hanseníase é uma doença crônica, infectocontagiosa, de evolução lenta e que acomete preferencialmente nervos e pele. A doença tem cura quando o tratamento é realizado de forma correta e sem interrupções, entretanto os indivíduos acometidos, se não tratados devidamente, podem apresentar sequelas, como incapacidades físicas ${ }^{(1,2)}$. $\mathrm{O}$ agente causador da hanseníase é o Mycobacterium leprae, que apresenta alta infectividade e baixa patogenicidade, ou seja, alto poder infectante e baixo poder de adoecimento, sendo transmitido de pessoa para pessoa pelo convívio diário com doentes contagiosos sem tratamento ${ }^{(3)}$.

Segundo a atualização global sobre hanseníase (2016), que tem como base as estatísticas anuais de 121 países, o número de casos novos registrados alcançou a soma de 213.899, com uma prevalência de 173.554. Um total de 18.869 novos pacientes detectados e notificados eram crianças e 1.312 recaídas foram notificadas em 46 países. Índia, Brasil e Indonésia notificam mais de dez mil novos pacientes anualmente, representando $81 \%$ dos pacientes recém-diagnosticados ${ }^{(4)}$.

O Brasil já postergou por duas vezes a meta de eliminação da hanseníase, primeiro em 2005 e outra em 2010. Uma nova meta foi estipulada para $2020^{(4)}$. E, apesar da intensificação das campanhas de combate à hanseníase, o país segue sendo o segundo país do mundo com maior número de casos novos de hanseníase, tendo nas regiões Norte e Nordeste as mais altas taxas de prevalência ${ }^{(5,6)}$. Em razão do estigma associado ao diagnóstico da hanseníase e seu alto poder incapacitante, a doença passou a ser considerada um problema de saúde pública, de forma que a sua eliminação constitui uma das atividades prioritárias do Ministério da Saúde (MS) desde o final do século XX ${ }^{(7-9)}$.

O tratamento preconizado pela Organização Mundial da Saúde (OMS) desde 1970 é realizado por meio da administração de antibióticos combinados, a poliquimioterapia (PQT), que é constituída por rifampicina, dapsona e clofazimina, com administração associada. Essa associação evita a resistência medicamentosa do bacilo, que ocorre com frequência quando se utiliza apenas um medicamento e impossibilita a cura da doença ${ }^{(2)}$.

Com a PQT, o tratamento de um caso de hanseníase compreende um esquema específico para as formas paucibacilares, que dura seis meses, e outro para os casos multibacilares, com duração de um ano, incluindo também o acompanhamento com vistas a identificar e tratar as possíveis intercorrências e complicações da doença, assim como a prevenção e o tratamento das incapacidades físicas. A alta por cura é dada após a administração do número de doses preconizadas pelo esquema terapêutico ${ }^{(1,2)}$.

O tratamento do paciente com hanseníase é essencial para interromper a cadeia de transmissão da doença, sendo, portanto, estratégico no controle da endemia e na eliminação da hanseníase enquanto problema de saúde pública. Por ter caráter longo, a adesão ao tratamento é essencial para o alcance da cura em hanseníase ${ }^{(2)}$.

Identifica-se a fragilidade na credibilidade quanto ao diagnóstico médico e a consequente inaceitação do uso de PQT como chave da política de eliminação da doença. Isso sugere a necessidade de uma maior atenção por parte dos serviços em saúde de trabalhar a orientação dos portadores, despertando a consciência de que a adesão e seguimento correto do tratamento trará a obtenção da cura da enfermidade ${ }^{(10)}$.

Na tentativa de alcançar as metas de eliminação da doença, o Brasil lançou o Programa Nacional de Controle da Hanseníase $(\mathrm{PNCH})$, que estabelece as bases e direciona as ações de vigilância em saúde para combate e eliminação dessa patologia(11). As atividades devem ser executadas no âmbito da Atenção Básica (AB), através da Estratégia Saúde da Família (ESF), por meio de ações que visam à promoção, prevenção, recuperação e reabilitação empenhada com a integralidade da assistência à saúde, tendo na família o foco das ações, observando o contexto socioeconômico, cultural e epidemiológico da comunidade em que está inserida ${ }^{(12)}$. 
A AB é responsável pelo acompanhamento do tratamento de hanseníase, uma estratégia que assegura o correto seguimento do tratamento. Na visita mensal ao posto de saúde, além da dispensação de medicamentos, é feita uma avaliação do paciente para acompanhar a evolução de suas lesões e o comprometimento neural, também são orientadas técnicas de autocuidado e prevenção de incapacidades e deformidades ${ }^{(2)}$.

O profissional enfermeiro atuante na $\mathrm{AB}$ possui papel extremamente importante na assistência dada ao paciente com diagnóstico de hanseníase ${ }^{(9)}$. Dentre as suas ações, deve-se destacar: detectar os portadores da forma contagiosa da doença na fase inicial do tratamento; inspecionar regularmente as lesões, principalmente nas extremidades; avaliar os contatos; informar ao paciente e aos familiares sobre a importância dos exercícios ativos e passivos dos membros acometidos; promover auxílio para aliviar os estigmas causados pela doença ${ }^{(1,13)}$.

Nessa perspectiva, é importante informar sobre a doença e a terapia ao paciente, à sociedade em geral e especialmente às famílias dos pacientes diagnosticados com hanseníase. A informação dos familiares a respeito da doença e seu compromisso no processo saúde/doença de seu familiar é um método eficaz para desmistificar o contágio. O apoio familiar é imprescindível para que o indivíduo aceite sua condição e não se sinta isolado nesse processo, colaborando para uma melhor adesão ao tratamento ${ }^{(14)}$. Dessa forma, o acolhimento é uma estratégia indispensável para fortalecer o vínculo com o usuário e os familiares, além de ser visto como uma medida relevante para o bom desempenho da rede assistencial do sistema de saúde.

A política de eliminação de hanseníase, portanto, exige do profissional enfermeiro o conhecimento para tomar decisões, orientar o planejamento de ações educativas, viabilizando intervenções compatíveis com as necessidades da comunidade ${ }^{(10)}$, o que faz este estudo necessário para uma efetiva promoção de saúde na AB. Assim, o objetivo deste trabalho foi avaliar a visão dos enfermeiros atuantes na Atenção Básica sobre aspectos do tratamento da hanseníase.

\section{MÉTODOS}

A pesquisa é do tipo descritiva, com abordagem qualitativa, realizada no município de Cocal, Piauí, Brasil, entre os meses de janeiro a março de 2016. As pesquisas qualitativas se caracterizam pela descrição e compreensão de um fenômeno, buscando a extrapolação para situações com contextos similares ${ }^{(15)}$, enquanto a pesquisa descritiva exige do investigador uma série de informações sobre o que deseja pesquisar. Esse tipo de estudo pretende descrever os fatos e fenômenos de determinada realidade ${ }^{(16)}$.

O município de Cocal está localizado no norte do estado, conta com 14 unidades de ESF compreendidas entre as zonas urbana e rural da cidade. Cada ESF é composta por um médico, um enfermeiro, um técnico de enfermagem e agentes comunitário de saúde (ACS).

Os profissionais enfermeiros foram objetos de estudo da presente pesquisa, sendo convidados a participar através de carta-convite e uma visita de um dos pesquisadores à Unidade de Saúde da Família, em que foram esclarecidos os objetivos da pesquisa. Incluíram-se profissionais enfermeiros com vínculo empregatício com a Secretaria Municipal de Saúde (SMS) de Cocal, Piauí, que desempenhassem atividades de assistência na AB do município e estivessem acessíveis no período da coleta de dados, confirmando sua participação no estudo por meio da assinatura do Termo de Consentimento Livre e Esclarecido (TCLE). Não fizeram parte da amostra enfermeiros que não faziam parte da $\mathrm{AB}$ do município ou desenvolvessem atividades administrativas na SMS, ou seja, não trabalhassem diretamente na assistência, executando ações na ESF. As entrevistas foram encerradas quando se identificou a saturação de dados, não sendo considerado relevante persistir na inclusão de novos participantes no estudo ${ }^{(17)}$. Dessa forma, a amostra final foi composta por nove enfermeiros.

As questões norteadoras aplicadas aos enfermeiros foram: Você sabe o que é o tratamento por poliquimioterapia (PQT)? Qual a sua influência na cura do paciente? Como se dá o tratamento do paciente com hanseníase? Para você qual a relevância da participação do enfermeiro? O que pode levar à falha do tratamento? O que isso pode causar ao paciente?

As entrevistas ocorreram em sala reservada da SMS para resguardo e sigilo das informações coletadas, em horário disponibilizado pelos profissionais, sem que comprometessem o cronograma de suas atividades junto à ESF. As entrevistas foram transcritas para efeito por meio da análise de conteúdo ${ }^{(18)}$, processo que visa ratificar o sentido das informações obtidas durante execução do trabalho por meio de artifícios de investigação que favorecem a comparação e explicação dos resultados.

A análise do conteúdo apresenta-se em três fases: pré-análise, fase em que se organiza o material a ser analisado com o objetivo de torná-lo operacional; exploração do material, em que há a definição de categorias (sistemas de codificação); e tratamento dos resultados, inferência e interpretação, etapa destinada ao tratamento dos resultados, em que ocorre a condensação e o destaque das informações para análise ${ }^{(18)}$.

Desta análise revelou-se a presença de três categorias temáticas: poliquimioterapia e cura; tratamento supervisionado; falha no tratamento.

A pesquisa recebeu aprovação no Comitê de Ética em Pesquisa do Hospital Eduardo de Menezes/HEM/FHEMIG sob o parecer $\mathrm{n}^{\mathrm{o}}$ 1.353.498. Para manter o anonimato, os entrevistados foram identificados pela letra E (Enfermeiro) mais o número respectivo da entrevista (E1 a E9). 


\section{RESULTADOS E DISCUSSÃO}

A seguir serão apresentadas as categorias temáticas que emergiram do estudo. Na primeira categoria, " poliquimioterapia e cura", foram agrupadas as falas que relacionam a cura da doença ao tratamento dispensado nas UBS; na categoria "Tratamento supervisionado" destaca-se a característica do acompanhamento longitudinal dispensado às pessoas atingidas por hanseníase; e na categoria "Falha no tratamento" são apontadas explicações para a falha terapêutica.

\section{Poliquimioterapia e cura}

Nesta categoria, os enfermeiros entrevistados reconheceram a PQT como eficaz para o tratamento da hanseníase, assim como para a redução da carga da doença para o sistema de saúde e para a cura do paciente, como se observa nos trechos abaixo:

"A PQT é uma combinação de antibióticos para matar as bactérias e evitar a resistência dos bacilos" (E1).

"O tratamento poliquimioterápico garante a redução da colonização do paciente e garante também a cura do paciente" (E5).

No que diz respeito à cura da hanseníase, os entrevistados destacam a importância da participação e comprometimento do paciente para que se possa atingi-la:

"A PQT nem sempre garante a cura do paciente, pois, às vezes, o paciente tem resistência a fazer o tratamento de forma enfocada" (E7).

"A medicação, se usada de forma correta, o paciente consegue a cura imediata" (E9).

Quando os enfermeiros foram questionados quanto ao conhecimento sobre o tratamento com a PQT e porque garante a cura do paciente, foram enfáticos ao reconhecerem a PQT como importante para o combate à hanseníase e consequentemente para a redução dos custos ao sistema de saúde, além de associarem seu uso correto com a cura da patologia.

A PQT foi instituída como tratamento da hanseníase a partir do ano de 1991, e desde então tem sido responsável pela queda na prevalência da doença. $\mathrm{O}$ tratamento constitui-se de uma combinação dos medicamentos (rifampicina, dapsona e clofazimina) e essa associação evita a resistência medicamentosa do bacilo, que ocorre frequentemente quando se utiliza apenas um desses compostos isoladamente. A PQT inviabiliza o bacilo, tornando-o incapaz de infectar outras pessoas; dessa forma, interrompe a cadeia epidemiológica da doença ${ }^{(1,2)}$.

As ações desenvolvidas pelas ESF visam o combate à hanseníase, objetivando sua erradicação. Seus integrantes devem atuar em conjunto na identificação de casos novos, acompanhando os pacientes em tratamento e nas orientações pós-alta ${ }^{(2,19)}$.

As orientações e esclarecimentos quanto ao uso correto da medicação e aos efeitos adversos que podem acontecer são pontos importantes que evitam o abandono do tratamento. Essas orientações cabem a todos os membros da ESF, dentre eles o enfermeiro. Nesse contexto, o conhecimento sobre o assunto é primordial para a execução de tais ações.

Uma pesquisa realizada em Teresina, Piauí, Brasil ${ }^{(20)}$ refletiu sobre a ação educativa que o enfermeiro desempenha na ESF junto ao portador de hanseníase, reafirmando seu papel de orientador em saúde. Além disso, chamou a atenção para a contribuição desse profissional através da consulta de enfermagem, sendo membro ativo na avaliação, no reconhecimento de sinais e sintomas, no planejamento e gerenciamento de ações que serão desenvolvidas com vistas ao combate à hanseníase.

Outrossim, os profissionais das UBS devem estar atentos para o surgimento das reações hansênicas, os efeitos adversos das medicações e o abandono do tratamento, sendo necessária agilidade nesses casos para que as devidas intervenções sejam realizadas $^{(21)}$. Entretanto, para que se alcance excelência nesse aspecto, deve haver uma boa conexão entre ESF e comunidade. O agente comunitário de saúde, considerado membro da ESF, funciona como elo entre a sociedade e o sistema de saúde, já que é quem possui contato mais próximo com a comunidade, observando de perto suas limitações e possibilidades ${ }^{(22)}$.

\section{Tratamento supervisionado}

Sobre essa categoria, os entrevistados ressaltaram a importância do acompanhamento, e associaram a supervisão ao tratamento correto, a garantia de cura, a redução de sequelas e a quebra da cadeia de transmissão:

"O tratamento supervisionado é essencial para garantir a cura e evitar a resistência do bacilo e a transmissão para outras pessoas" (E1).

“(...) porque é necessário assegurar que a medicação esteja sendo tomada corretamente, minimizar as sequelas e incapacidades” (E5).

“(...) principalmente porque uma vez que o paciente para de usar a medicação, o paciente corre o risco de recidivar e ficar mais resistente à medicação” (E9).

As ações dispensadas na supervisão do tratamento pelo profissional enfermeiro foram destacadas:

"No encontro mensal, administram-se as doses supervisionadas, faz-se a avaliação física das lesões e suas complicações, observa-se se há resistência ao tratamento" (E2). 
“A supervisão é eficaz para que o profissional de saúde esteja consciente da correta tomada da medicação pelo paciente em domicilio, obtendo, assim, êxito na cura do mesmo” (E3).

"Avalia-se as possíveis reações medicamentosas, avalia-se lesões, avalia-se os contatos, os nervos periféricos, uma vez que a doença que pode causar incapacidade física” (E8).

Sobre o acompanhamento dos pacientes em tratamento da hanseníase e sua importância, os entrevistados afirmaram tal medida ser essencial para a cura e tratamento da patologia. Os enfermeiros citaram que o acompanhamento é importante para que se certifiquem de que a medicação está sendo tomada da forma adequada e para evitar o contágio de outras pessoas. A supervisão foi destacada como ferramenta útil para verificar o surgimento de efeitos adversos, incapacidades físicas e avaliação dos contatos. Esse aspecto reflete a consciência dos profissionais, uma vez inseridos na ESF, para com seu papel transformador junto à comunidade $\mathrm{e}^{(10,23)}$.

A portaria GM 2.488/2011 do MS cita que o papel do enfermeiro, além de educar e prevenir, é participar do tratamento através da consulta de enfermagem, solicitando exames complementares, coordenando e avaliando as ações desenvolvidas na $\mathrm{ESF}^{(24)}$.

Um estudo realizado em Sobral, Ceará, Brasil( ${ }^{(25)}$, analisou a percepção dos enfermeiros e portadores de hanseníase sobre a consulta de enfermagem, além de identificar as dificuldades vivenciadas durante a execução da consulta de enfermagem no decorrer do tratamento dos portadores de hanseníase. Valendo-se de uma abordagem subjetiva, os autores concluíram que os enfermeiros buscam excelência na execução de seus serviços, apesar de grande demanda; criação de vínculo, para um tratamento humanizado; e prioridade de cura e prevenção de incapacidades.

A AB é um cenário voltado para o desenvolvimento de ações que promovam a saúde dos usuários, individual ou coletivamente. $\mathrm{O}$ enfermeiro é um dos articuladores dessa promoção, no que se refere à hanseníase suas ações contribuem para diminuir a incidência e para o controle da doença, através do exame dermatoneurológico, avaliação de sinais sintomas e direcionamento do tratamento. Uma das dificuldades apontadas na literatura é que a demanda elevada de pessoas associada à responsabilidade gerencial pela UBS influenciam no tempo da consulta de enfermagem, sendo um pouco mais rápida do que deveria ser e influenciando negativamente na assistência prestada à população ${ }^{(21,23)}$.

O acompanhamento do paciente com hanseníase deve ser continuado após a alta e completar o esquema da PQT, chamado de momento pós-alta. Os pacientes podem apresentar reações hansênicas e/ou recidivas, as primeiras responsáveis pelo comprometimento neural e por gerar incapacidades físicas nos seus portadores ${ }^{(26)}$. No entanto, as reações hansênicas podem surgir durante o tratamento e levar o paciente ao abandono do dele se não forem bem orientados.

Pesquisa $^{(27)}$ sobre aspectos da assistência de enfermagem às reações hansênicas evidenciou a importância do profissional enfermeiro no cuidado aos pacientes com hanseníase em crise reacional, identificação da neurite silenciosa pela palpação dos nervos periféricos e observação da coloração e aspecto das manchas que podem vir a se manifestar na pele dos indivíduos. Os autores destacam que, além da consulta de enfermagem, o enfermeiro realiza exames, avaliações e encaminhamentos na procura precoce dos achados dermatoneurológicos, tendo um amplo escopo de ações ${ }^{(27)}$.

\section{Falha no tratamento}

Essa categoria associa a falha do tratamento, principalmente quanto ao engajamento do paciente e da família, falta de medicação no posto de saúde, falta de orientação ao paciente, ao nível de escolaridade e aos efeitos colaterais dos remédios:

"O que está muito associado à falha do tratamento é a falta de compromisso do paciente e sua familia, falta de medicação e acompanhamento pela ESF" (E2).

"Vários fatores podem levar à falha do tratamento, como falta de cuidadores e falta de medicamentos" (E7).

"Os efeitos das drogas podem levar o paciente à desistência" (E5).

“(...) baixa escolaridade, falta de cobertura do ESF/ACS, alcoolismo, falta de reavaliação, descompromisso do paciente” (E8).

A não adesão e não correto seguimento do tratamento foram relacionados às complicações, como agravamento de sintomas, incapacidades, amputações e até ao óbito:

"Paciente não tratado pode causar todas as complicações da doença, como comprometimentos dos nervos, amputação, etc, e continuar a transmissão da doença” (E7).

"Pode provocar a não cura, recidiva e resistência" (E6).

"A recidiva da doença, incapacidade física e até a morte do paciente podem acontecer se o tratamento não for feito direitinho" (E2).

"A não aderência ao tratamento pode causar incapacidades físicas" (E8).

"Se o tratamento não for seguido corretamente, pode levar à morte desse paciente, pois afeta partes importantes que comprometem à saúde” (E9). 
Quando indagados sobre o que pode ocasionar a falha do tratamento e suas repercussões no paciente, os participantes da presente pesquisa enumeram pontos interessantes, como o compromisso do paciente para com o tratamento, a falta de medicação, os efeitos colaterais da medicação, falta de cuidadores, baixa escolaridade, falta de cobertura pela ESF, entre outros. Esses são temas de uma realidade local, mas que podem ser comparados aos de outras regiões.

A baixa escolaridade dificulta o acesso à informação e proporciona a compreensão errônea de determinados conceitos. Os esclarecimentos devem ser transmitidos com o objetivo de se fazer entender pelo paciente, atendendo as suas necessidades, utilizando uma linguagem acessível ${ }^{(28)}$.

Uma pesquisa desenvolvida no Vale do Jequitinhonha, Minas Gerais, Brasil(29), buscou conhecer as ações desenvolvidas pelos serviços de saúde e as estratégias empregadas para fazer frente ao controle da hanseníase baseadas no diagnóstico precoce, tratamento oportuno, prevenção e tratamento de incapacidade e vigilância dos contatos. Essas ações são os principais eixos na atenção à hanseníase na Atenção Básica ${ }^{(2)}$.

As ações de educação em saúde desenvolvidas na Atenção Básica são de suma importância para combate à hanseníase, mesmo com essa relevância comprovada cientificamente, os enfermeiros ainda se mantêm presos ao modelo de atendimento clínico assistencial, muitas vezes sendo observadas ações de combate quando já há instalação do quadro. Uma das razões para a ocorrência desse fato deve-se ao grande número de programas que compõem a $\mathrm{AB}$ e são de responsabilidade do enfermeiro ${ }^{(30-33)}$.

Além disso, aponta-se que uma maior cobertura da ESF está intimamente relacionada à redução dos focos de contágio da hanseníase e contribui para prevenir incapacidades físicas e promover adesão ao tratamento ${ }^{(34)}$. Acredita-se, assim, que a estrutura e organização dos serviços de saúde têm grande influência na situação epidemiológica da hanseníase, fator considerado, inclusive, mais dominante do que os fatores socioeconômicos. Essas informações corroboram os achados deste estudo, sobretudo quando os entrevistados apontam a falta de medicamentos a serem dispensados na terapêutica da hanseníase, apontando para uma falha gerencial do $\mathrm{PCNH}$, ou mesmo da saúde municipal ${ }^{(35)}$.

Os efeitos adversos da medicação, como o escurecimento da pele, devem-se à Clofazimina, que é tomada uma vez por mês e também diariamente para o tratamento multibacilar. A alteração desaparece dentro de alguns meses depois do término do tratamento, mas, enquanto visível, contribui para a estigmatização da doença. Além disso, a presença de reações hansênicas, que são reações do sistema imunológico do doente ao bacilo, apresentam-se como a principal causa de lesões dos nervos e de incapacidades provocadas pela hanseníase, representando um grande problema no tratamento dos portadores ${ }^{(2,36)}$.

Em pesquisa ${ }^{(37)}$ realizada em Governador Valadares, Minas Gerais, Brasil, com pacientes com diagnóstico de hanseníase em 2011, o relato dos entrevistados voltado às reações da poliquimioterapia cita a mudança da cor da pele e dos lábios, dores de cabeça e de estômago, entre outras. Tais reações causam preocupação aos pacientes, especialmente as expostas a olho nu, devido à dificuldade em responder aos questionamentos das pessoas sobre as mencionadas reações ${ }^{(37)}$.

O estigma da hanseníase é bastante associado pela literatura como causador de falha no tratamento da hanseníase. Estudos mostram que os pacientes ainda sofrem com medo e preconceito que o diagnóstico proporciona, e esse fato pode levar ao diagnóstico tardio e abandono do tratamento ${ }^{(28)}$. A identificação oportuna dos sinais e sintomas e o esclarecimento sobre a doença e o tratamento levam o paciente a sentir confiança e dar seguimento ao tratamento ${ }^{(29)}$, momento em que a UBS têm função primordial.

Observa-se, no presente estudo, que a maior preocupação dos enfermeiros participantes foi com a PQT, dando pouca ênfase à relevância da participação da família como coadjuvante no apoio ao familiar com diagnóstico de hanseníase. Esse achado condiz com estudo realizado ${ }^{(38)}$ no município de São Gonçalo, na região metropolitana do Rio de Janeiro, Brasil, em 2009, cujo objetivo foi identificar quais as ações realizadas pelo enfermeiro do Programa de Saúde da Família (PSF) para detectar a hanseníase e qual o cuidado por ele oferecido às pessoas atingidas pela doença tendo como foco a humanização.

A prática da educação em saúde na assistência ao usuário do programa de hanseníase deve ser entendida como prática transformadora, a ser desenvolvida pela equipe de saúde em ações de controle da hanseníase e envolvendo usuários, familiares e comunidade. Assim, o cuidado de enfermagem deve ser prestado de forma integral, atendendo o indivíduo em todas as suas necessidades biológicas, sociais, psicológicas e culturais; por isso é necessário que o usuário do programa de hanseníase tenha um cuidado supervisionado que contribua para reduzir o abandono do tratamento e aumente o número de pessoas curadas ${ }^{(1,39)}$.

O lado social do paciente com diagnóstico de hanseníase deve ser visto com cuidado, de modo que ele possa prosseguir com suas atividades diárias na sociedade e na família, longe de estigmas e preconceitos.

O estudo teve como limitação a não identificação de assuntos intrínsecos que dificultam o tratamento da hanseníase na Atenção Básica (infraestrutura, dificuldade no acesso à medicação, entre outros), pois foram utilizadas perguntas norteadoras muito abrangentes. Sugerem-se novas pesquisas com vistas a esclarecer de forma pormenorizada os empecilhos que dificultam o acesso à informação por parte dos pacientes sobre a hanseníase e para ouvir não só os profissionais enfermeiros, mas também os demais integrantes da ESF e usuários.

\section{CONCLUSÃO}

O estudo mostrou que os enfermeiros voluntários possuem informações básicas coerentes às informações veiculadas pelo MS sobre o tratamento de hanseníase. As entrevistas evidenciaram ainda aspectos que devem ser corrigidos para que se 
alcance excelência no combate à hanseníase, como o pouco compromisso do paciente com seu tratamento e a dificuldade de acompanhamento da ESF.

Este estudo torna-se importante para que, a partir do reconhecimento desses pontos deficientes, possam ser traçadas medidas de controle. Reforça-se que as estratégias de educação em saúde devem ser estimuladas na prevenção da hanseníase, no incentivo para participação dos familiares como apoio ao tratamento do paciente, na adesão dos pacientes ao tratamento e no esclarecimento de preconceitos estabelecidos, diminuindo os estigmas que o diagnóstico de hanseníase proporciona.

\section{REFERÊNCIAS}

1. Ministério da Saúde (BR), Secretaria de Atenção à Saúde, Departamento de Atenção Básica. Vigilância em Saúde: Dengue, Esquistossomose, Hanseníase, Malária, Tracoma e Tuberculose. $2^{\mathrm{a}}$ ed. rev. Brasília: Ministério da Saúde; 2008.

2. Ministério da Saúde (BR), Secretaria de Políticas de Saúde. Departamento de Atenção Básica. Guia para o Controle da hanseníase. Brasília: Ministério da Saúde; 2002.

3. Portal da Sociedade Brasileira de Dermatologia. Hanseníase [acesso em 2016 Mar 16]. Disponível em: http://www.sbd.org. br/doencas/hanseniase/

4. Organização Mundial da Saúde. Estratégia global para hanseníase 2016-2020: aceleração rumo a um mundo sem hanseníase. Genebra: OMS; 2016.

5. Zanardo TS, Santos SM, Oliveira VCC, Mota RM, Mendonça BOM, Nogueira DS, et al. Perfil epidemiológico dos pacientes com hanseníase na atenção básica de saúde de São Luís de Montes Belos, no período de 2008 a 2014. Rev Faculdade Montes Belos. 2016;9(2):77-90.

6. Sousa AA, Oliveira FJF, Costa ACPJ, Santos M Neto, Cavalcante EFO, Ferreira AGN. Adesão ao tratamento da hanseníase por pacientes acompanhados em unidades básicas de saúde de Imperatriz-MA. SANARE (Sobral). 2013;12(1):6-12.

7. Ministério da Saúde (BR). Portaria $\mathrm{n}^{\circ} 3.125$, de 7 de outubro de 2010. Aprova as diretrizes para vigilância, atenção e controle da hanseníase [acesso em 2016 Mar 16].. Disponível em: http://bvsms.saude.gov.br/bvs/saudelegis/gm/2010/ prt3125_07_10_2010.html

8. Oliveira LP, Alves HJ, Silva MA. Construindo estratégias para o enfrentamento da hanseníase no âmbito da atenção básica/ primária: apontamentos para o trabalho do enfermeiro. In: Anais do $11^{\circ}$ Congresso Internacional da Rede Unida. Rev Interface. 2014 (Supl).

9. Cabral CVS, Costa MAO, Lima RBO, Silva JS, Cabral LC, Rocha NMC. O papel do enfermeiro na prevenção de incapacidades e deformidades no portador de hanseníase. Rev Interdisciplinar. 2016;9(2):168-77.

10. Tavares LI, Pinheiro BE, Santos AMD, Costa PN. Adesão ao tratamento da Hanseníase: dificuldades inerentes aos portadores. Rev Bras Enferm. 2010;63(6):983-90.

11. Lanza FM, Vieira NF, Oliveira MMC, Lana FCF. Avaliação da atenção primária no controle da hanseníase: proposta de uma ferramenta destinada aos usuários. Rev Esc Enferm USP. 2014;48(6):1054-61.

12. Ministério da Saúde (BR), Secretaria de Atenção à Saúde, Departamento de Atenção Básica. Política Nacional de Atenção Básica. Brasília : Ministério da Saúde; 2012.

13. Duarte LMCPS, Simpson CA, Silva TMS, Moura IBL, Isoldi DMR. Ações de autocuidado de pessoas com hanseníase. Rev Enferm UFPE. 2014;8(8):2816-22.

14. Minuzzo DA. O homem paciente de Hanseníase (Lepra): representação social, rede social familiar, experiência e imagem corporal [dissertação]. Évora: Universidade de Évora; 2008.

15. Ollaik LG, Ziller HM. Concepções de validade em pesquisas qualitativas. Educação Pesquisa. 2012;38(1):229-41.

16. Triviños ANS. Introdução à pesquisa em ciências sociais: a pesquisa qualitativa em educação. São Paulo: Atlas; 1987.

17. Fontanella BJB, Ricas J, Turato ER. Amostragem por saturação em pesquisas qualitativas em saúde: contribuições teóricas. Cad. Saúde Pública. 2008;24(1):17-27.

18. Bardin L. Análise de conteúdo. Lisboa: Edições 70; 1977.

19. Moura LMA, Pereira MA, Veloso LC. Estratégias utilizadas pelos serviços de saúde na detecção precoce da hanseníase: uma revisão integrativa. Rev Saúde Foco. 2015;2(1):130-50.

20. Reis MF, Albuquerque KR, Silva MP, Nascimento FCV, Paiva MP. Vivência de enfermeiros da atenção básica nas ações de controle da hanseníase no município de Teresina-PI. Rev Saúde Foco. 2015;2(2):115-24. 
21. Silva RP, Beserra EP, Nunes EM, Alves ESRC, Gomes MR. Consulta de enfermagem em atenção primária ao portador de hanseníase: proposta de instrumento. Arq Ciênc Saúde. 2015;22(1):38-32.

22. Sales JCS, Luz VLES, Branco FMFC, Araujo MFF, Castro SHTS, Silva TCA. O significado da hanseníase para o agente comunitário de saúde. Rev Interdisciplinar. 2013;6(1):17-24.

23. Piovesan LR, Schimith MD, Simon BS, Budó MLD, Weiller TH, Brêtas ACP. Promoção da saúde na perspectiva de enfermeiros de atenção básica. Rev Enferm UERJ. 2016;24(3):e5816.

24. Ministério da Saúde (BR). Portaria no 2.488/2011. Aprova a Política Nacional de Atenção Básica, estabelecendo a revisão de diretrizes e normas para a organização da Atenção Básica, para a Estratégia Saúde da Família (ESF) e o Programa de Agentes Comunitários de Saúde (PACS) [acesso em 2017 Fev 22]. Disponível em: http://bvsms.saude.gov.br/bvs/ saudelegis/gm/2011/prt2488_21_10_2011.html

25. Freitas CASL, Silva AV Neto, Ximenes FRG Neto, Albuquerque IMN, Cunha ICKO. Consulta de enfermagem ao portador de hanseníase no território da estratégia saúde da família: percepções de enfermeiro e pacientes. Rev Bras Enferm. 2008;61(esp):757-63.

26. Ribeiro MDA, Oliveira SB, Filgueiras MC. Pós-alta em hanseníase: uma revisão sobre qualidade de vida e conceito de cura. Saúde (Santa Maria). 2015;41(1):9-18.

27. Paschoal VDA, Soler ZASG. O fenômeno reacional na hanseníase e aspectos da assistência de enfermagem. REFACS (Online). 2015;3(1):46-51.

28. Lana FCF, Lanza FM, Carvalho APM, Tavares APN. O estigma em hanseníase e sua relação com as ações de controle. Rev Enferm UFSM. 2014;4(3):556-65.

29. Lanza FM, Lana FCF, Carvalho APM, Davi RFL. Ações de controle da hanseníase: tecnologias desenvolvidas nos municípios do vale do Jequitinhonha, Minas Gerais. Rev Enferm Cent.-Oeste Min. 2011;1(2):164-75.

30. Aguiar PG, Almeida DA, Silva SDC, Paschoini J. Fatores de manutenção da endemia hansênica e as ações da enfermagem no controle da hanseníase. Rev Iniciação Científica Libertas. 2014;4(1):119-32.

31. Coêlho LS, Albuquerque KR, Maia NMFS, Carvalho LRB, Almeida CAPL, Silva MP. Vivência do enfermeiro da atenção básica nas ações de controle da hanseníase. Rev Enferm UFPE. 2015;9(Supl 10):1411-7.

32. Silva LSR, Silva TM, Rocha JT, Andrade WG, Lessa EC, Correia NS. A assistência de enfermagem aos portadores de hanseníase assistidos pelo programa de saúde da família. Rev Enferm UFPE. 2016;10(11): 4111-7.

33. Ferreira VF, Rocha GOR, Lopes MMB, Santos MS, Miranda SA. Educação em saúde e cidadania: revisão integrativa. Trab Educ Saúde. 2014;12(2):363-78.

34. Andrade MV, Noronha KVMS, Menezes RM, Souza MN, Reis CB, Martins DR, et al. Desigualdade socioeconômica no acesso aos serviços de saúde no Brasil: um estudo comparativo entre as regiões brasileiras em 1998 e 2008 . Econ Apl. 2013;17(4):623-45.

35. Amaral EP, Lana FCF. Análise espacial da Hanseníase na microrregião de Almenara, MG, Brasil. Rev Bras Enferm. 2008; 61(esp.):701-7.

36. Organização Mundial da Saúde. Estratégia global aprimorada para redução adicional da carga da hanseníase: 2011-2015, diretrizes operacionais (atualizadas). Brasília: Organização Pan-Americana da Saúde; 2010.

37. Silveira MGB, Coelho AR., Rodrigues SM, Soares MM, Camillo GN. Portador de hanseníase: impacto psicológico do diagnóstico. Psicol Soc. 2014;26(2):517-27.

38. Santos PN, Zerbinato PHM, Mota Silva A, Rodrigues DP, Oliveira LS, Antunes Cortez E, et al. La detección de la lepra y la humanización de la atención: acciones de enfermería en el programa de salud de la família. Enferm Glob. 2012;11(1):10415.

39. Ministério da Saúde (BR). Hanseníase e direitos humanos: direitos e deveres dos usuários do SUS. Brasília: Ministério da Saúde; 2008.

\author{
Endereço para correspondência: \\ Mara Dayanne Alves Ribeiro \\ R. Cel. Estanislau Frota, s/n \\ Bairro: Centro \\ CEP: 62010-560 - Sobral - CE - Brasil \\ E-mail: fisiomaraday@gmail.com
}

